\title{
PTH-133 A REAL LIFE RETROSPECTIVE ANALYSIS OF DRUG EXPENDITURE REVEALS NO SIGNIFICANT COST DIFFERENCE BETWEEN INFLIXIMAB AND ADALIMUMAB IN THE TREATMENT OF ADULT CROHN'S DISEASE
}

doi:10.1136/gut.2011.239301.534

C A Lamb, ${ }^{1, *}$ M Price, ${ }^{2}$ M Robinson, ${ }^{2}$ M C Gunn, ${ }^{2}$ N P Thompson, $^{2}$ J C Mansfield ${ }^{1}{ }^{1}$ Institute of Cellular Medicine, Newcastle University, Newcastle upon Tyne, UK; ${ }^{2}$ Gastroenterology, Newcastle upon Tyne Hospitals NHS Foundation Trust, Newcastle upon Tyne, UK

Introduction The revised NICE guidance on the use of infliximab and adalimumab for the treatment of Crohn's disease, released in May 2010, has suggested biologics be prescribed as a planned therapy until treatment failure or until completion of 12 months treatment, whichever is shorter. For the first time, NICE have introduced cost as a criteria for which anti-TNF antibody treatment should be used, recommending that therapy be commenced with the less expensive of the two drugs. However the comparative cost of the drugs is not immediately clear. For example the dosage of infliximab is weight dependent, whereas the dosage of adalimumab is independent of weight; and the dosage interval of both drugs may be shortened to optimise efficacy. These factors may impact on cost.

Methods As part of a service development exercise, a retrospective audit of patient records was performed. The drug cost of infliximab and adalimumab prescription per patient for the treatment of Crohn's disease in the Trust between 1 April 2009 and 31 March 2010 was calculated.

Results Of 60 patients receiving biologics, 46 patients were treated with infliximab and 19 patients with adalimumab during the 1-year audit period (5 patients received both drugs during 


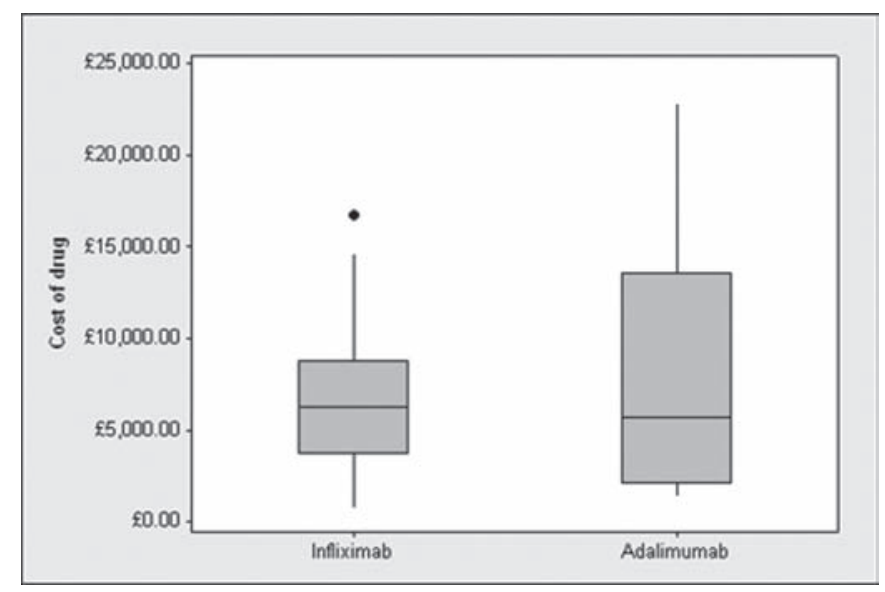

Figure 1 PTH-133 Cost of infliximab and adalimumab per patient between 1 April 2009 and 31 March 2010.

the study period). The mean age (range) of the cohort was 32.4 years (17-65). The median cost of infliximab was £6294 per patient (mean (95\% CI) cost, £6877 (5783 to 7971) per patient). The median cost of adalimumab was $£ 5720$ per patient (mean (95\% CI) cost, £8226 (4893 to 11560 ) per patient). There was no significant cost difference between the drugs ( $p=0.43$ ).

Conclusion In this cohort of patients, no significant cost difference was found when comparing infliximab and adalimumab for the treatment of Crohn's disease over a 1-year period. In light of the recent NICE guidance, this data would support individual patient and physician choice when deciding on which anti-TNF therapy to prescribe.

Competing interests None.

Keywords adalimumab, anti-TNF therapy, cost, Crohn's disease, infliximab. 